\title{
Synthesis of Unnatural 1-Methyl-2-quinolone Derivatives
}

\author{
Motoki Asahara, Taku Katayama, Yasuo Tohda, Nagatoshi Nishiwaki, ${ }^{*}$ and Masahiro Ariga* \\ Department of Chemistry, Osaka Kyoiku University; 4-698-1 Asahigaoka, Kashiwara, Osaka 582-8582, Japan. \\ Received August 9, 2004; accepted September 1, 2004; published online September 2, 2004
}

Unnatural 1-methyl-2-quinolone derivatives were synthesized by regioselective $\mathbf{C}-\mathrm{C}$ bond formation. When 1-methyl-3,6,8-trinitro-2-quinolone (TNQ) was treated with enamines, nucleophilic addition readily occurred at the 4-position, and succeeding hydrolysis of enamine moiety followed by elimination of nitrous acid furnished 4acylmethyl-1-methyl-6,8-dinitro-2-quinolones. The same products could be prepared by the reaction of TNQ with ketones in the presence of triethylamine. The present reaction enabled the introduction of various kinds of acylmethyl groups substituted with alkyl, aryl or hetaryl groups.

Key words 1-methyl-2-quinolone; trinitroquinolone; cine-substitution; regioselective $\mathrm{C}-\mathrm{C}$ bond formation

The 1-methyl-2-quinolone (MeQone) skeleton has been found in more than 300 quinoline alkaloids those are mostly isolated from the Rutaceae family. ${ }^{1-15)}$ Since these alkaloids show physiological activities, many researchers have energetically studied the isolation, the structural determination and total syntheses of quinoline alkaloids containing the MeQone skeleton. ${ }^{4-15)}$ From the viewpoint for the drug design, it is also demanded to synthesize hitherto unknown unnatural MeQone derivatives and to develop new methods for functionalization of the MeQone framework. ${ }^{16-32)}$ Especially, modification of the pyridone moiety in the MeQone is highly important because most of the naturally occurring MeQones have substituents at the 3 and/or the 4-position. Mainly used methods for functionalization of the MeQone involve the activation by the pre-introduced substituents such as hydroxyl, alkoxyl and amino groups, ${ }^{4-15)}$ which compose partial structures of newly constructed skeleton. Meanwhile, direct functionalization methods of MeQone were recently attracted. ${ }^{16-29)}$ As one of the methodologies, Fujita and co-workers prepared phenanthridine derivatives by Diels-Alder reaction of electron-rich dienes with MeQone having an electron-withdrawing group at the 3 or 4-position. ${ }^{30-32)}$

In our course of study on electron-deficient quinolones, 1methyl-3,6,8-trinitro-2-quinolone (TNQ) was found to be highly reactive, which realized direct functionalization of the MeQone. ${ }^{33)}$ The steric repulsion between the 8-nitro and the 1-methyl groups activates the pyridone ring of TNQ, which

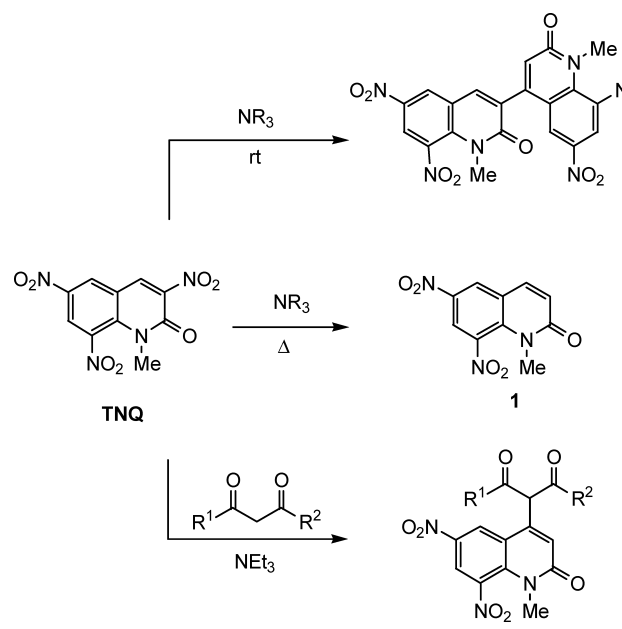

reveals nitroalkene property rather than aromatic one. ${ }^{34)}$ When TNQ was allowed to react with tertiary amine in acetonitrile, dimer of TNQ connected at the 3- and 4'-positions was obtained at room temperature, and denitration on the pyridone ring occurred at the elevated temperature leading to $1{ }^{35)}$ On the other hand, 4-functionalized 6,8-dinitro-2quinolones, were readily formed upon treatment of TNQ with 1,3-dicarbonyl compounds in the presence of triethylamine, in which regioselective $\mathrm{C}-\mathrm{C}$ bond formation at the 4position is achieved. ${ }^{33)}$ Although the present cine-substitution is useful for direct functionalization of the quinolone ring, only 1,3-dicarbonyl compounds were usable. Thus, it is one of the important projects to enable the employment other nucleophiles, which provides a new methodology for the quinolone chemistry. Since we succeed to introduce a variety of acylmethyl groups at the 4-position of the MeQone skeleton, results will be represented in this paper.

\section{Results and Discussion}

Enamines were employed as the carbon nucleophiles instead of 1,3-dicarbonyl compounds. When TNQ was treated with 1-morpholino-1-phenylethene $\mathbf{2 a}$ in the presence of water at room temperature, 4-benzoylmethyl-6,8-dinitro-2quinolone 3a was isolated (Table 1, run 1). This reaction was considerably affected by steric hindrance at the $\beta$-position of enamines, namely the presence of substituent $R^{2}$. When enamines $\mathbf{2 b}$ and $\mathbf{2 c}$ were employed, morpholinium salts $\mathbf{4 b}$ and $\mathbf{4 c}$ were obtained instead of cine-substituted products $\mathbf{3 b}$ and $\mathbf{3 c}$ (runs 2 and 3). Cyclic enamine $\mathbf{2 d}$ also reacted with

Table 1. Reactions of TNQ with Enamines

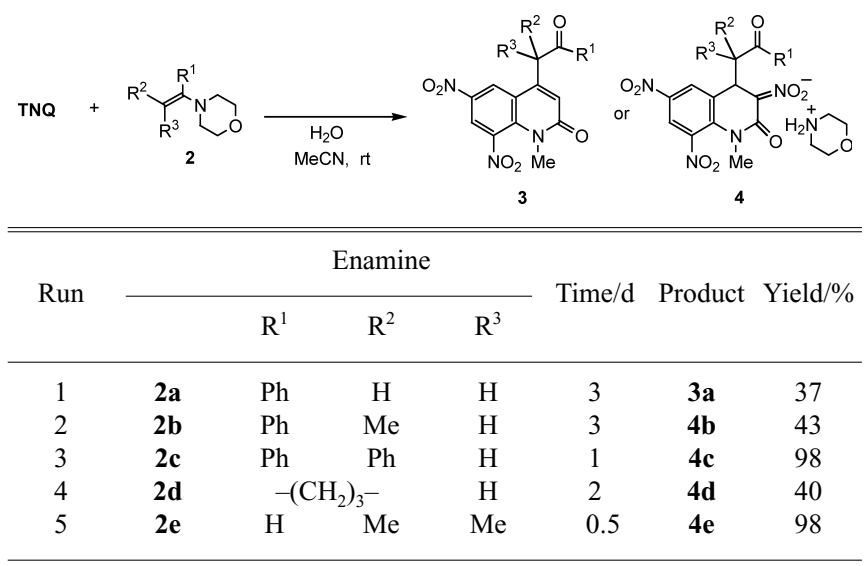

Chart 1. Functionalized MeQones derived from TNQ 

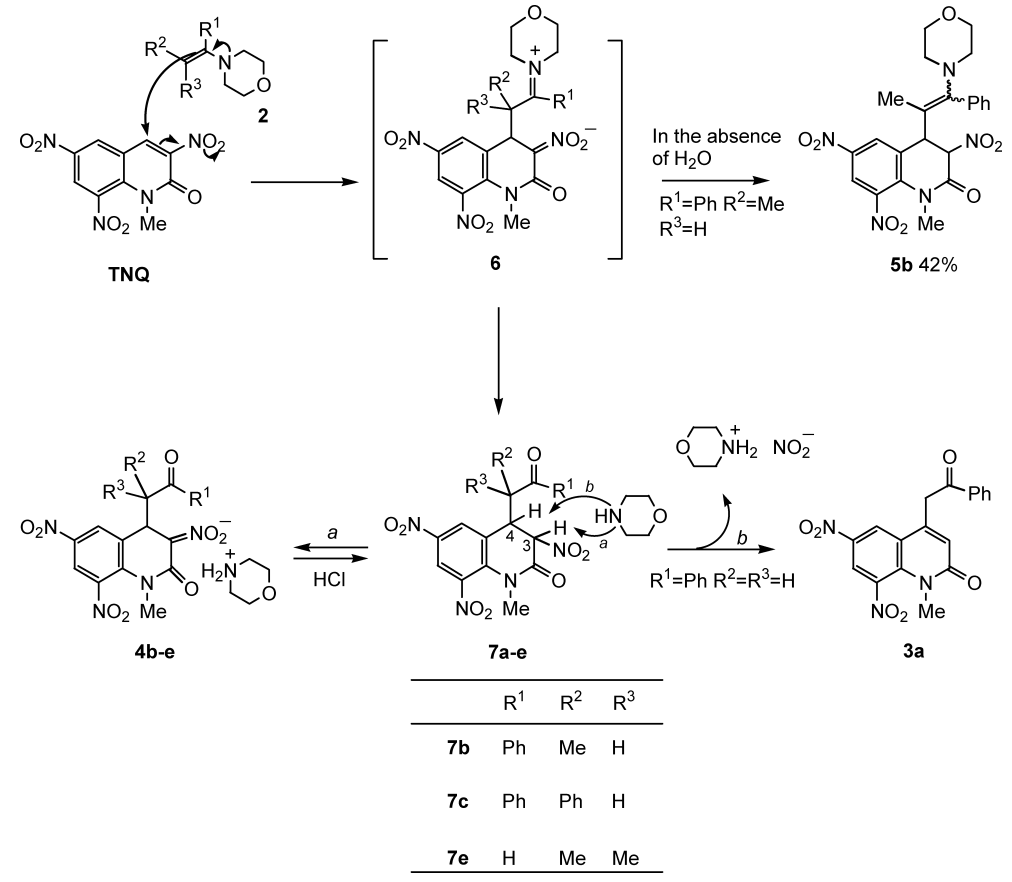

Chart 2. A Plausible Mechanism for Reactions of TNQ with Enamines

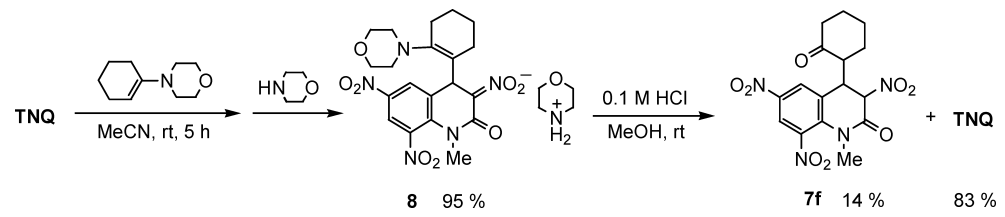

Chart 3. Trap of Meisenheimer Complex as Morpholinium Nitronate

TNQ to give corresponding salt $\mathbf{4 d}$ (run 4). Enamine $\mathbf{2 e}$ derived from aldehyde was more reactive leading to salt $\mathbf{4 e}$ in an excellent yield within short reaction time even though $\mathbf{2 e}$ had two methyl groups at the reaction site (run 5).

A plausible mechanism for these reactions of TNQ with enamines is illustrated in Chart 2. An enamine attacks at the electron deficient 4-position of TNQ giving Meisenheimer complex 6. The following hydrolysis of the immonium moiety leads to 3,4-dihydroquinolone 7, and morpholine is liberated during this process. Deprotonation at the 3-position by morpholine gives morpholinium salt $\mathbf{4}$. On the other hand, deprotonation at the 4-position causes elimination of nitrous acid to afford cine-substituted product 3 . When substituent at the 4-position of dihydroquinolone 7 is sterically hindered, the former reaction is preferable than the latter one.

Intermediate dihydroquinolone $\mathbf{5 b}$ was isolated in the reaction conducted under dried conditions to avoid hydrolysis. The result strongly supported our proposed addition-elimination mechanism for cine-substitution. ${ }^{33)}$ While it was somewhat difficult to isolate adduct 5 because of hydrolysis, effective trap of $\mathbf{5}$ as morpholinium salt $\mathbf{8 f}$ was achieved by addition of extra morpholine to the reaction mixture. Dihydroquinolone $7 \mathbf{f}$ could be obtained by acidification of $8 \mathbf{f}$ despite in a low yield, and the major part of $\mathbf{8 f}$ was converted to TNQ with elimination of the ketone moiety at the 4-position. Dihydroquinolones $\mathbf{7 b}, \mathbf{7} \mathbf{c}$ and $7 \mathbf{e}$ were respectively isolated in good yields when morpholinium salts $\mathbf{4 b}, \mathbf{4 c}$ and $4 \mathbf{e}$ were acidified with hydrochloric acid.
Results mentioned so far prompted us to employ less reactive ketones as the nucleophiles. In this reaction, addition of base seemed to be necessary for accelerating enolization of ketones 9 and for assisting elimination of nitrous acid from intermediate dihydroquinolone 7 . When TNQ was treated with acetophenone 9a in the presence of triethylamine, cinesubstitution readily proceeded to afford 4-(benzoylmethyl)quinolone 3a in a good yield. The present reaction was applicable to other ketones, and results were summarized in Table 2 and Chart 4 . This reaction was applicable to $\alpha$ monosubstituted acetophenones 9b and 9c (Table 2, runs 2 and 3), however $\alpha, \alpha$-disubstituted acetophenone 9l caused no change (run 5). Bicyclic ketone, tetralone $\mathbf{9 m}$, also reacted with TNQ affording $\mathbf{3 m}$ in a moderate yield (run 6). Aliphatic ketones 9d and $\mathbf{9 f}-\mathbf{j}$ showed similar reactivity to afford corresponding 4-substituted dinitroquinolones $\mathbf{3 d}$ and 3f-j (runs 7-10 and Chart 4). When unsymmetrical butanone $9 \mathrm{~h}$ was used, thermodynamically controlled enol was more reactive than kinetically controlled one, and leading to 3h as a major product. The yield of cine-substituted product was considerably lowered in the case of 3-pentanone $\mathbf{9 j}$ because of steric hindrance, and the competitive addition of triethylamine at the 4-position was preferred, which caused denitration to give 6,8-dinitroquinolone 1 (run 10). ${ }^{35)}$ Aldehyde $9 \mathbf{e}$ was reactive to afford cine-substituted product $\mathbf{3 e}$ despite the presence of two methyl groups at the reaction site. Furthermore, the present reaction realized the introduction of hetaroylmethyl groups to the MeQone skeleton. 2- 
Table 2. cine-Substitution of TNQ with Ketones

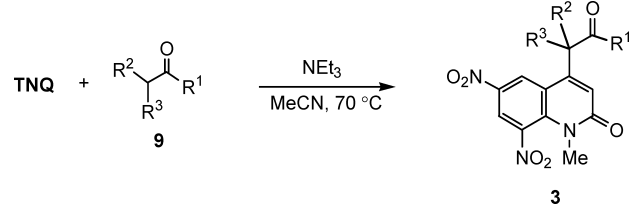

\begin{tabular}{|c|c|c|c|c|c|c|}
\hline \multirow{2}{*}{ Run } & \multicolumn{4}{|c|}{ Ketone } & \multirow{2}{*}{ Time/h } & \multirow{2}{*}{ Yield $/ \%$} \\
\hline & & $\mathrm{R}^{1}$ & $\mathrm{R}^{2}$ & $\mathrm{R}^{3}$ & & \\
\hline 1 & $9 \mathbf{a}$ & $\mathrm{Ph}$ & $\mathrm{H}$ & $\mathrm{H}$ & 2.5 & 83 (3a) \\
\hline 2 & $9 b$ & $\mathrm{Ph}$ & $\mathrm{Me}$ & $\mathrm{H}$ & 3.5 & $77(\mathbf{3 b})$ \\
\hline 3 & $9 \mathrm{c}$ & $\mathrm{Ph}$ & $\mathrm{Ph}$ & $\mathrm{H}$ & 3.5 & $69(3 c)$ \\
\hline 4 & $9 \mathbf{k}$ & $4-\mathrm{MeC}_{6} \mathrm{H}_{4}$ & $\mathrm{H}$ & $\mathrm{H}$ & 2.5 & 59 (3k) \\
\hline 5 & 91 & $\mathrm{Ph}$ & $\mathrm{Me}$ & $\mathrm{Me}$ & 6 & $0^{a)}(31)$ \\
\hline 6 & $9 m$ & \multicolumn{2}{|c|}{$-\left(o-\mathrm{C}_{6} \mathrm{H}_{4}\right) \mathrm{CH}_{2} \mathrm{CH}_{2}-$} & $\mathrm{H}$ & 2 & $52(3 \mathrm{~m})$ \\
\hline 7 & 9d & \multicolumn{2}{|c|}{$-\left(\mathrm{CH}_{2}\right)_{3}-$} & $\mathrm{H}$ & 2 & $58(\mathbf{3 d})$ \\
\hline 8 & $9 f$ & \multicolumn{2}{|c|}{$-\left(\mathrm{CH}_{2}\right)_{4}-$} & $\mathrm{H}$ & 2 & $82(3 f)$ \\
\hline 9 & $9 \mathrm{~g}$ & $\mathrm{Me}$ & $\mathrm{H}$ & $\mathrm{H}$ & 3.5 & $83(3 \mathbf{g})$ \\
\hline 10 & $9 j$ & Et & $\mathrm{Me}$ & $\mathrm{H}$ & 4 & $18^{a)}(\mathbf{3} \mathbf{j})$ \\
\hline 11 & $9 e$ & $\mathrm{H}$ & $\mathrm{Me}$ & $\mathrm{Me}$ & 5 & $41(3 \mathbf{e})$ \\
\hline 12 & $9 n$ & 2-Pyridyl & $\mathrm{H}$ & $\mathrm{H}$ & 2.5 & 74 (3n) \\
\hline 13 & 90 & 2-Furyl & $\mathrm{H}$ & $\mathrm{H}$ & 3 & $45(30)$ \\
\hline
\end{tabular}

a) 6,8-Dinitroquinolone 1 was isolated in $41 \%$ (run 5) and $73 \%$ (run 10) yields.
TNQ

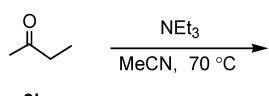

$9 \mathrm{~h}$

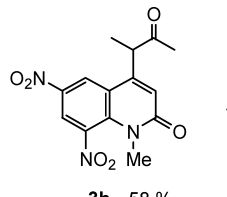

3h $58 \%$

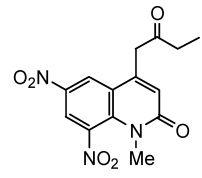

3i $9 \%$
Chart 4. Reaction of TNQ with 2-Butanone $\mathbf{9 h}$

Acetylpyridine 9n and 2-acetylfuran 9o showed similar reactivity to furnish dihydroquinolones $\mathbf{3 n}$ and $\mathbf{3 o}$ (runs 12 and 13).

In summary, TNQ was shown to be an excellent precursor for unnatural MeQone derivatives having an acylmethyl group 3 and their related compounds. Enamines readily reacted with TNQ at room temperature to give 4-acylmethylquinolones 3, which were formed by hydrolysis of adduct 6 and dihydroquinolones 7. Furthermore, ketones were usable for this reaction as the nucleophile instead of enamines though the reaction should be conducted in the presence of triethylamine at higher temperature. In the present reaction, aliphatic or alicyclic ketones could be used, and introduction of acylmethyl or 2-oxocycloalkyl groups was achieved at the 4-position of 6,8-dinitroquinolones. Aroylmethyl and hetaroylmethyl groups could be also introduced by using aryl and hetaryl ketones.

These reactions required only simple experimental manipulations, and the $\mathrm{C}-\mathrm{C}$ bond formation at the 4-position of the MeQone skeleton was performed regioselectively. Hence, this reaction would provide a new methodology for functionalization of the MeQone skeleton.

\section{Experimental}

General The melting points were determined on a Yanaco micro-melting-points apparatus, and were uncorrected. ${ }^{1} \mathrm{H}-\mathrm{NMR}$ spectra were measured on a Bruker DPX-400 at $400 \mathrm{MHz}$ and on a Hitachi R1200 at $60 \mathrm{MHz}$ with TMS as an internal standard. ${ }^{13} \mathrm{C}$-NMR spectra were measured on a Bruker DPX-400 at $100 \mathrm{MHz}$ with TMS as an internal standard, and assignments of signals $(\mathrm{s}, \mathrm{d}, \mathrm{t}$ and $\mathrm{q}$ ) were made from DEPT experiments. IR spectra were recorded on a Horiba FT-200 IR spectrometer. Elemental microanalyses were performed using a Yanaco MT-3 CHN corder. All the reagents and solvents were commercially available and used as received. All of reactions were carried out under ambient atmosphere. Column chromatography was performed using Wakogel C-200.

1-Methyl-3,6,8-trinitro-2-quinolone (TNQ) Following the procedure described for 1-methyl-2-pyridone, ${ }^{36)}$ MeQone was prepared by oxidation of 1-methylquinolinium ion using potassium ferricyanide (III) under alkaline conditions after methylation of quinoline with dimethyl sulfate using the three times diluted solution. Nitration of MeQone with fuming nitric acid $(d=1.52)$ afforded TNQ in $90 \%$ yield. $\left.^{33}\right)$

Typical Procedure for the Reaction of TNQ with Enamines in the Presence of Water To a solution of TNQ $(0.28 \mathrm{~g}, 0.95 \mathrm{mmol})$ and water $(0.85 \mathrm{~g}, 47.2 \mathrm{mmol})$ in acetonitrile $(25 \mathrm{ml})$, was added a solution of 1 -morpholino-1-phenylethene $\mathbf{2 a}(0.26 \mathrm{~g}, 1.37 \mathrm{mmol})$ in acetonitrile $(10 \mathrm{ml})$ at room temperature, and the mixture was stirred for $3 \mathrm{~d}$. Generated precipitates were collected by filtration to afford 4-benzoylmethyl-6,8-dinitro-1methyl-2-quinolone (3a) as pale yellow powder $(0.13 \mathrm{~g}, 0.35 \mathrm{mmol}, 37 \%)$ mp 229-230 ${ }^{\circ} \mathrm{C}$ (dec.). IR $\left(\mathrm{KBr} / \mathrm{cm}^{-1}\right) 1682,1670,1529,1342 ;{ }^{1} \mathrm{H}-\mathrm{NMR}$ $\left(400 \mathrm{MHz}, \mathrm{DMSO}-d_{6}\right) \delta: 3.37(\mathrm{~s}, 3 \mathrm{H}), 5.05(\mathrm{~s}, 2 \mathrm{H}), 6.98(\mathrm{~s}, 1 \mathrm{H}), 7.62(\mathrm{dd}$, $J=7.2,7.4 \mathrm{~Hz}, 2 \mathrm{H}), 7.73(\mathrm{t}, J=7.4 \mathrm{~Hz}, 1 \mathrm{H}), 8.13(\mathrm{~d}, J=7.2 \mathrm{~Hz}, 2 \mathrm{H}), 8.71(\mathrm{~d}$, $J=2.5 \mathrm{~Hz}, 1 \mathrm{H}), 8.89$ (d, $J=2.5 \mathrm{~Hz}, 1 \mathrm{H}) ;{ }^{13} \mathrm{C}-\mathrm{NMR}\left(100 \mathrm{MHz}, \mathrm{DMSO}-d_{6}\right) \delta$ : 34.4 (q), 41.8 (t), 122.2 (d), 123.6 (s), 124.9 (d), 125.1 (d), 128.4 (d), 128.7 (d), 133.7 (d), 135.9 (s), 137.6 (s), 138.7 (s), 139.9 (s), 145.5 (s), 161.2 (s), 195.9 (s). Anal. Calcd for $\mathrm{C}_{18} \mathrm{H}_{13} \mathrm{~N}_{3} \mathrm{O}_{6}$ : C, 58.85; H, 3.57; N, 11.44. Found: C, 58.67; H, 3.45; N, 11.50 .

Reactions of TNQ with other enamines were conducted in the same way.

Morpholinium 4-(1-Benzoylethyl)-3,4-dihydro-6,8-dinitro-1-methyl-2oxoquinoline-3-nitronate (4b) Pale yellow powder. $\mathrm{mp} 160-162^{\circ} \mathrm{C}$ (dec.). IR ( $\left.\mathrm{KBr} / \mathrm{cm}^{-1}\right) 1675,1657,1537,1522,1358,1336 ;{ }^{1} \mathrm{H}-\mathrm{NMR}$ $\left(400 \mathrm{MHz}, \mathrm{CDCl}_{3}\right) \delta: 1.06(\mathrm{~d}, J=6.7 \mathrm{~Hz}, 3 \mathrm{H}), 1.2-2.5(\mathrm{br}, 2 \mathrm{H}), 3.03(\mathrm{~s}$, $3 \mathrm{H}), 3.2-3.4(\mathrm{~m}, 4 \mathrm{H}), 3.9-4.1(\mathrm{~m}, 4 \mathrm{H}), 4.2-4.3(\mathrm{~m}, 1 \mathrm{H}), 5.16(\mathrm{~d}$, $J=3.6 \mathrm{~Hz}, 1 \mathrm{H}), 7.51(\mathrm{~m}, 2 \mathrm{H}), 7.63(\mathrm{t}, J=7.5 \mathrm{~Hz}, 1 \mathrm{H}), 7.92(\mathrm{~d}, J=2.2 \mathrm{~Hz}$, $1 \mathrm{H}), 8.16(\mathrm{~d}, J=8.0 \mathrm{~Hz}, 2 \mathrm{H}), 8.44(\mathrm{~d}, J=2.2 \mathrm{~Hz}, 1 \mathrm{H}) ;{ }^{13} \mathrm{C}-\mathrm{NMR}(100 \mathrm{MHz}$, $\left.\mathrm{CDCl}_{3}\right) \delta: 12.3(\mathrm{q}), 34.8(\mathrm{q}), 43.2(\mathrm{~d}), 43.8(\mathrm{t}), 45.3(\mathrm{~d}), 64.1(\mathrm{t}), 108.0(\mathrm{~s})$, 120.5 (d), 126.7 (d), 128.7 (d), 129.0 (d), 131.5 (s), 133.7 (s), 136.1 (s), 138.6 (s), 141.0 (s), 162.9 (s), 200.6 (s). Anal. Calcd for $\mathrm{C}_{23} \mathrm{H}_{25} \mathrm{~N}_{5} \mathrm{O}_{9}: \mathrm{C}$, 53.59; H, 4.89; N, 13.59. Found: C, 53.39; H, 4.88; N, 13.72 .

Morpholinium 4-( $\alpha$-Benzoylbenzyl)-3,4-dihydro-6,8-dinitro-1-methyl2-oxoquinoline-3-nitronate (4c) Yellow powder. mp $132-133^{\circ} \mathrm{C}$ (dec.). IR $\left(\mathrm{KBr} / \mathrm{cm}^{-1}\right) 1684,1651,1535,1371,1335 ;{ }^{1} \mathrm{H}-\mathrm{NMR}\left(400 \mathrm{MHz}, \mathrm{CDCl}_{3}\right)$ $\delta: 2.31(\mathrm{~s}, 3 \mathrm{H}), 3.26-3.29(\mathrm{~m}, 4 \mathrm{H}), 3.96-3.99(\mathrm{~m}, 4 \mathrm{H}), 4.97(\mathrm{~d}, J=2.3 \mathrm{~Hz}$, $1 \mathrm{H}), 5.56(\mathrm{~d}, J=2.3 \mathrm{~Hz}, 1 \mathrm{H}), 6.84(\mathrm{dd}, J=6.4,3.4 \mathrm{~Hz}, 2 \mathrm{H}), 7.11-7.12(\mathrm{~m}$, $3 \mathrm{H}), 7.25-7.46(\mathrm{~m}, 3 \mathrm{H}), 7.92(\mathrm{~d}, J=7.4 \mathrm{~Hz}, 2 \mathrm{H}), 8.42(\mathrm{~d}, J=2.6 \mathrm{~Hz}, 1 \mathrm{H})$, $9.03(\mathrm{~d}, J=2.6 \mathrm{~Hz}, 1 \mathrm{H}) ;{ }^{13} \mathrm{C}-\mathrm{NMR}\left(100 \mathrm{MHz} \mathrm{CDCl}_{3}\right) \delta: 34.1(\mathrm{q}), 42.0(\mathrm{~d})$, 43.5 (t), 57.8 (d), 63.8 (t), 109.1 (s), 120.3 (d), 127.9 (d), 128.5 (d), 128.6 (d), 128.9 (d), 129.1 (d), 129.2 (d), 131.8 (s), 133.1 (d), 135.3 (s), 135.9 (s), 138.5 (s), 139.6 (s), 141.1 (s), 162.7 (s), 198.2 (s). Anal. Calcd for $\mathrm{C}_{28} \mathrm{H}_{27} \mathrm{~N}_{5} \mathrm{O}_{9}$ : C, 58.22; H, 4.71; N, 12.13. Found: C, 58.04; H, 4.57; N, 11.94 .

Morpholinium 3,4-Dihydro-6,8-dinitro-1-methyl-4-(2-oxocyclopentyl)-2-oxoquinoline-3-nitronate (4d) Yellow powder. $\mathrm{mp} 126$ $128^{\circ} \mathrm{C}$ (dec.). IR ( $\left.\mathrm{KBr} / \mathrm{cm}^{-1}\right) 1732,1660,1525,1338 ;{ }^{1} \mathrm{H}-\mathrm{NMR}(400 \mathrm{MHz}$, $\left.\mathrm{CDCl}_{3}\right) \delta: 1.41-1.44(\mathrm{~m}, 1 \mathrm{H}), 1.76-1.82(\mathrm{~m}, 1 \mathrm{H}), 1.94-2.04(\mathrm{~m}, 3 \mathrm{H})$, $2.26-2.32(\mathrm{~m}, 1 \mathrm{H}), 2.54-2.59(\mathrm{~m}, 1 \mathrm{H}), 3.13(\mathrm{~s}, 3 \mathrm{H}), 3.27$ (brt, $J=4.6 \mathrm{~Hz}$, 4H), 3.98 (brt, $J=4.6 \mathrm{~Hz}, 4 \mathrm{H}), 5.14$ (d, $J=4.7 \mathrm{~Hz}, 1 \mathrm{H}), 8.29$ (d, $J=2.5 \mathrm{~Hz}$, $1 \mathrm{H}), 8.49(\mathrm{~d}, J=2.5 \mathrm{~Hz}, 1 \mathrm{H}) ;{ }^{13} \mathrm{C}-\mathrm{NMR}\left(100 \mathrm{MHz} \mathrm{CDCl}_{3}\right) \delta: 20.4(\mathrm{t}), 26.0$ $(\mathrm{t}), 34.5(\mathrm{q}), 38.1(\mathrm{t}), 39.3(\mathrm{~d}), 43.6(\mathrm{t}), 53.4(\mathrm{~d}), 64.0(\mathrm{t}), 108.0(\mathrm{~s}), 120.4(\mathrm{~d})$, 126.7 (d), 132.8 (s), 138.7 (s), 139.4 (s), 141.4 (s), 162.8 (s), 217.1 (s). Anal. Calcd for $\mathrm{C}_{19} \mathrm{H}_{23} \mathrm{~N}_{5} \mathrm{O}_{9}$ : C, 49.03; H, 4.98; N, 15.05. Found: C, 49.43; H, 5.10; N, 14.93.

Morpholinium 3,4-Dihydro-4-(1,1-dimethyl-2-oxoethyl)-6,8-dinitro-1methyl-2-oxoquinoline-3-nitronate (4e) Yellow powder. $\mathrm{mp} 175-177^{\circ} \mathrm{C}$ (dec.). IR (KBr/ $\left.\mathrm{cm}^{-1}\right)$ 1718, 1659, 1543, 1524, 1338; ${ }^{1} \mathrm{H}-\mathrm{NMR}(400 \mathrm{MHz}$, $\left.\mathrm{CDCl}_{3}\right) \delta: 0.78(\mathrm{~s}, 3 \mathrm{H}), 0.97(\mathrm{~s}, 3 \mathrm{H}), 2.87(\mathrm{~s}, 3 \mathrm{H}), 3.00-3.15(\mathrm{~m}, 4 \mathrm{H})$, $3.2-4.0(\mathrm{br}, 2 \mathrm{H}), 3.75-3.76(\mathrm{~m}, 4 \mathrm{H}), 4.96(\mathrm{~s}, 1 \mathrm{H}), 8.39(\mathrm{~s}, 1 \mathrm{H}), 8.57(\mathrm{~s}$, $1 \mathrm{H}), 9.48(\mathrm{~s}, 1 \mathrm{H}) ;{ }^{13} \mathrm{C}-\mathrm{NMR}\left(100 \mathrm{MHz}, \mathrm{CDCl}_{3}\right) \delta: 17.5$ (q), 19.3 (q), 33.5 (q), $43.2(\mathrm{t}), 44.8(\mathrm{~d}), 52.4(\mathrm{t}), 63.6(\mathrm{~s}), 102.8(\mathrm{~s}), 120.4$ (d), 126.9 (d), 131.5 (s), 137.7 (s), 139.7 (s), 140.06 (s), 161.2 (s), 203.7 (d). Anal. Calcd for $\mathrm{C}_{18} \mathrm{H}_{23} \mathrm{~N}_{5} \mathrm{O}_{9}$ : C, 47.68; H, 5.11; N, 15.45. Found: C, 47.55; H, 5.16; N, 15.51 .

3,4-Dihydro-1-methyl-4-(1-morpholino-1-phenyl-2-propenyl)-3,6,8trinitro-2-oxoquinoline (5b) Pale yellow needles. mp $157-158^{\circ} \mathrm{C}$ (dec.). IR $\left(\mathrm{KBr} / \mathrm{cm}^{-1}\right) 1718,1653,1566,1541,1344 ;{ }^{1} \mathrm{H}-\mathrm{NMR}\left(60 \mathrm{MHz}, \mathrm{CDCl}_{3}\right) \delta$ : 
$1.83(\mathrm{~s}, 3 \mathrm{H}), 2.73(\mathrm{t}, J=4.5 \mathrm{~Hz}, 4 \mathrm{H}), 3.14(\mathrm{~s}, 3 \mathrm{H}), 3.73(\mathrm{t}, J=4.5 \mathrm{~Hz}, 4 \mathrm{H})$ $4.33(\mathrm{~d}, J=13.0 \mathrm{~Hz}, 1 \mathrm{H}), 5.56(\mathrm{~d}, J=13.0 \mathrm{~Hz}, 1 \mathrm{H}), 6.9-7.5(\mathrm{~m}, 5 \mathrm{H}), 8.42$ $(\mathrm{d}, J=2.4 \mathrm{~Hz}, 1 \mathrm{H}) 8.62(\mathrm{~d}, J=2.4 \mathrm{~Hz}, 1 \mathrm{H})$.

Morpholinium 3,4-Dihydro-6,8-dinitro-1-methyl-4-(2-morpholino-2,3cyclohexenyl)-2-oxoquinoline-3-nitronate (8f) To a solution of TNQ $(0.60 \mathrm{~g}, 2.04 \mathrm{mmol})$ in acetonitrile $(40 \mathrm{ml})$, was added a solution of 1-morpholino-1-cyclohexene $2 \mathrm{f}(0.66 \mathrm{~g}, 3.95 \mathrm{mmol})$ and morpholine $(0.18 \mathrm{~g}$, $2.07 \mathrm{mmol})$ in acetonitrile $(10 \mathrm{ml})$, and the resultant mixture was stirred at room temperature for $4 \mathrm{~h}$. Morpholinium salt $8 \mathbf{f}$ was precipitated as orange powder during the reaction, and was collected by filtration $(1.05 \mathrm{~g}$, $1.93 \mathrm{mmol}, 95 \%) \mathrm{mp} 143-145^{\circ} \mathrm{C}$ (dec.). IR $\left(\mathrm{KBr} / \mathrm{cm}^{-1}\right) 1653,1539,1333$; ${ }^{1} \mathrm{H}-\mathrm{NMR}\left(400 \mathrm{MHz}, \mathrm{CDCl}_{3}\right) \delta: 0.95-0.98(\mathrm{~m}, 1 \mathrm{H}), 1.16-1.25(\mathrm{~m}, 2 \mathrm{H})$, $1.61-2.00(\mathrm{~m}, 3 \mathrm{H}), 2.60-2.75(\mathrm{~m}, 2 \mathrm{H}), 3.11(\mathrm{~s}, 3 \mathrm{H}), 3.00-3.30(\mathrm{~m}, 1 \mathrm{H})$ $3.17-3.30(\mathrm{~m}, 4 \mathrm{H}), 3.31-3.51(\mathrm{~m}, 2 \mathrm{H}), 3.80-4.03(\mathrm{~m}, 8 \mathrm{H}), 5.05(\mathrm{brt}$, $J=3.0 \mathrm{~Hz}, 1 \mathrm{H}), 5.16(\mathrm{~d}, J=3.0 \mathrm{~Hz}, 1 \mathrm{H}), 8.45(\mathrm{~d}, J=2.3 \mathrm{~Hz}, 1 \mathrm{H}), 8.48(\mathrm{~d}$, $J=2.3 \mathrm{~Hz}, 1 \mathrm{H}) ;{ }^{13} \mathrm{C}-\mathrm{NMR}\left(100 \mathrm{MHz}, \mathrm{CDCl}_{3}\right) \delta: 21.1(\mathrm{t}), 24.7(\mathrm{t}), 25.0(\mathrm{t})$, 34.9 (q), 38.7 (d), 41.2 (d), 44.6 (t), $49.4(\mathrm{t}), 65.3$ (t), 67.3 (t), 109.4 (d), 109.5 (s), 119.9 (d), 127.8 (d), 129.3 (s), 138.3 (s), 139.7 (s), 141.0 (s), 144.6 (s), 163.4 (s). Anal. Calcd for $\mathrm{C}_{24} \mathrm{H}_{32} \mathrm{~N}_{6} \mathrm{O}_{9} \cdot \mathrm{CH}_{3} \mathrm{CN}$ : C, 52.96; H, 5.98 ; $\mathrm{N}, 16.63$. Found: C, 52.96; H, 6.10; N, 16.65 .

3,4-Dihydro-1-methyl-4-(2-oxocyclohexyl)-3,6,8-trinitro-2-quinolone (7f) To a solution of morpholinium salt $8 \mathbf{8 f}(0.38 \mathrm{~g}, 0.693 \mathrm{mmol})$ in methanol $(30 \mathrm{ml}), 1 \mathrm{~m}$ hydrochloric acid $(1.4 \mathrm{ml}, 1.4 \mathrm{mmol})$ was added, and the mixture was stirred for $15 \mathrm{~min}$. Generated precipitates were collected by filtration, and were washed with methanol $(15 \mathrm{ml})$ to give a mixture of isomeric $7 \mathbf{f}$ and TNQ as white powder $(0.21 \mathrm{~g}$, trans-7f, $10 \%$, cis-7f, $4 \%$, TNQ, $83 \%$ yields, These values were determined by ${ }^{1} \mathrm{H}-\mathrm{NMR}$ ). trans-7f ${ }^{1} \mathrm{H}-\mathrm{NMR}$ $\left(400 \mathrm{MHz}, \mathrm{CDCl}_{3}\right) \delta: 1.20-2.50(\mathrm{~m}, 8 \mathrm{H}), 2.84-2.90(\mathrm{~m}, 1 \mathrm{H}), 3.30(\mathrm{~s}$, $3 \mathrm{H}), 4.49(\mathrm{dd}, J=2.1,8.0 \mathrm{~Hz}, 1 \mathrm{H}), 5.85(\mathrm{~d}, J=2.1 \mathrm{~Hz}, 1 \mathrm{H}), 8.62(\mathrm{~d}$, $J=2.6 \mathrm{~Hz}, 1 \mathrm{H}), 8.66(\mathrm{~d}, J=2.6 \mathrm{~Hz}, 1 \mathrm{H}) . c i s-7 \mathrm{f}^{1} \mathrm{H}-\mathrm{NMR}\left(400 \mathrm{MHz}, \mathrm{CDCl}_{3}\right)$ $\delta: 1.20-2.50(\mathrm{~m}, 8 \mathrm{H}), 3.00-3.06(\mathrm{~m}, 1 \mathrm{H}), 3.19(\mathrm{~s}, 3 \mathrm{H}), 4.55(\mathrm{dd}, J=1.8$ $6.1 \mathrm{~Hz}, 1 \mathrm{H}), 5.94(\mathrm{~d}, J=1.8 \mathrm{~Hz}, 1 \mathrm{H}), 8.65(\mathrm{~s}, 2 \mathrm{H})$.

4-(1-Benzoylethyl)-3,4-dihydro-1-methyl-3,6,8-trinitro-2-quinolone (7b) To a solution of morpholinium salt $4 \mathbf{b}(0.47 \mathrm{~g}, 1.0 \mathrm{mmol})$ in methanol $(30 \mathrm{ml}), 1 \mathrm{~m}$ hydrochloric acid $(1.5 \mathrm{ml}, 1.5 \mathrm{mmol})$ was added, and the mixture was stirred for $3 \mathrm{~d}$. Generated precipitates were collected by filtration, and were washed with methanol $(15 \mathrm{ml})$ to give $7 \mathbf{b}$ as white powder $(0.27 \mathrm{~g}, 69 \%$ yield). $\mathrm{mp} 140.5-141.5^{\circ} \mathrm{C}$ (dec.). IR $\left(\mathrm{KBr} / \mathrm{cm}^{-1}\right) 1689,1653,1568,1545$, 1384; ${ }^{1} \mathrm{H}-\mathrm{NMR}\left(400 \mathrm{MHz}, \mathrm{CDCl}_{3}\right) \delta: 1.32(\mathrm{~d}, J=7.2 \mathrm{~Hz}, 1 \mathrm{H}), 3.30(\mathrm{~s}, 3 \mathrm{H})$, $3.64(\mathrm{dq}, J=9.0,7.2 \mathrm{~Hz}, 1 \mathrm{H}), 4.42(\mathrm{dd}, J=9.0,2.2 \mathrm{~Hz}, 1 \mathrm{H}), 5.34(\mathrm{~d}$, $J=2.2 \mathrm{~Hz}, 1 \mathrm{H}), 7.49-7.63(\mathrm{~m}, 2 \mathrm{H}), 7.65(\mathrm{t}, J=6.3 \mathrm{~Hz}, 1 \mathrm{H}), 7.84-7.86(\mathrm{~m}$, $2 \mathrm{H}), 8.38(\mathrm{~d}, J=2.6 \mathrm{~Hz}, 1 \mathrm{H}), 8.63(\mathrm{~d}, J=2.6 \mathrm{~Hz}, 1 \mathrm{H}) ;{ }^{13} \mathrm{C}-\mathrm{NMR}(100 \mathrm{MHz}$, $\mathrm{CDCl}_{3}$ ) $\delta: 17.4$ (q), 34.8 (q), 41.3 (d), 44.2 (d), 84.3 (d), 121.8 (d), 127.9 (d), 128.4 (d), 128.7 (s), 128.9 (s), 129.3 (d), 134.6 (d), 134.7 (s), 138.6 (s), 142.7 (s), 155.6 (s), 199.4 (s). Anal. Calcd for $\mathrm{C}_{19} \mathrm{H}_{16} \mathrm{~N}_{4} \mathrm{O}_{8}$ : C, 53.27; $\mathrm{H}$, 3.77; N, 13.08. Found: C, 52.99; H, 3.68; N, 12.90 .

Acidification of $4 \mathbf{c}$ and $4 \mathbf{e}$ was performed in a similar way.

4-( $\alpha$-Benzoylbenzyl)-3,4-dihydro-1-methyl-3,6,8-trinitro-2-quinolone (7c) Reaction time $1 \mathrm{~d}, 97 \%$ yield. White powder. mp $143-144^{\circ} \mathrm{C}$ (dec.). IR $\left(\mathrm{KBr} / \mathrm{cm}^{-1}\right) 1684,1653,1574,1549,1342 ;{ }^{1} \mathrm{H}-\mathrm{NMR}\left(400 \mathrm{MHz}, \mathrm{CDCl}_{3}\right)$ $\delta: 3.12(\mathrm{~s}, 3 \mathrm{H}), 4.58(\mathrm{~d}, J=8.3 \mathrm{~Hz}, 1 \mathrm{H}), 4.86(\mathrm{dd}, J=8.3,2.0 \mathrm{~Hz}, 1 \mathrm{H}), 5.48$ $(\mathrm{d}, J=2.0 \mathrm{~Hz}, 1 \mathrm{H}), 6.93-6.95(\mathrm{~m}, 2 \mathrm{H}), 7.23-7.26(\mathrm{~m}, 3 \mathrm{H}), 7.35-7.39(\mathrm{~m}$, $2 \mathrm{H}), 7.51(\mathrm{t}, J=7.4 \mathrm{~Hz}, 1 \mathrm{H}), 7.82-7.83(\mathrm{~m}, 2 \mathrm{H}), 7.85(\mathrm{~d}, J=2.6 \mathrm{~Hz}, 1 \mathrm{H})$, $8.50(\mathrm{~d}, J=2.6 \mathrm{~Hz}, 1 \mathrm{H}) ;{ }^{13} \mathrm{C}-\mathrm{NMR}\left(100 \mathrm{MHz}, \mathrm{CDCl}_{3}\right) \delta: 34.5(\mathrm{q}), 44.3(\mathrm{~d})$, 54.5 (d), 85.0 (d), 121.4 (d), 128.4 (d), 128.5 (s), 128.6 (d), 128.9 (d), 129.0 (d), 129.3 (d), 129.8 (d), 133.5 (s), 134.2 (s), 134.9 (s), 138.5 (s), 139.8 (s), 142.3 (s), 159.3 (s), 195.2 (s). Anal. Calcd for $\mathrm{C}_{24} \mathrm{H}_{18} \mathrm{~N}_{4} \mathrm{O}_{8}$ : C, 58.77; $\mathrm{H}$, 3.70 ; N, 11.43. Found: C, 58.81; H, 3.70; N, 11.32.

3,4-Dihydro-4-(1,1-dimethyl-2-oxoethyl)-1-methyl-3,6,8-trinitro-2quinolone (7e) Reaction time $12 \mathrm{~h}, 99 \%$ yield. Pale yellow needles. $\mathrm{mp}$ $173-175^{\circ} \mathrm{C}$ (dec.). IR $\left(\mathrm{KBr} / \mathrm{cm}^{-1}\right) 1720,1653,1560,1541,1344 ;{ }^{1} \mathrm{H}-\mathrm{NMR}$ $\left(400 \mathrm{MHz}, \mathrm{CDCl}_{3}\right) \delta: 1.15(\mathrm{~s}, 3 \mathrm{H}), 1.21(\mathrm{~s}, 3 \mathrm{H}), 3.24(\mathrm{~s}, 3 \mathrm{H}), 4.33(\mathrm{~d}$, $J=1.6 \mathrm{~Hz}, 1 \mathrm{H}), 5.43(\mathrm{~d}, J=1.6 \mathrm{~Hz}, 1 \mathrm{H}), 8.35(\mathrm{~d}, J=2.5 \mathrm{~Hz}, 1 \mathrm{H}), 8.63(\mathrm{~d}$, $J=2.5 \mathrm{~Hz}, 1 \mathrm{H}), 9.41(\mathrm{~s}, 1 \mathrm{H}) ;{ }^{13} \mathrm{C}-\mathrm{NMR}\left(100 \mathrm{MHz}, \mathrm{CDCl}_{3}\right) \delta: 19.7$ (q), 20.2 (q), 34.8 (q), 45.8 (d), 48.2 (s), 83.7 (d), 109.3 (s), 121.9 (s), 125.9 (d), 127.5 (s), 128.9 (d), 139.2 (s), 160.0 (s), 200.8 (s). Anal. Calcd for $\mathrm{C}_{14} \mathrm{H}_{14} \mathrm{~N}_{4} \mathrm{O}_{8}$ : C, $45.90 ; \mathrm{H}, 3.85 ; \mathrm{N}, 15.30$. Found: C, 46.21; H, 3.84; N, 15.04 .

6,8-Dinitro-1-methyl-4-\{2-(4-methylphenyl)-2-oxoethyl\}-2-quinolone (3k) To a solution of TNQ $(0.29 \mathrm{~g}, 0.99 \mathrm{mmol})$ and 4-methylacetophenone $(6.95 \mathrm{~g}, 51.80 \mathrm{mmol})$ in acetonitrile $(15 \mathrm{ml})$, was added a solution of triethylamine $(0.20 \mathrm{~g}, 1.98 \mathrm{mmol})$ in acetonitrile $(5 \mathrm{ml})$, and the resultant mixture was heated at $70^{\circ} \mathrm{C}$ for $2.5 \mathrm{~h}$. After removal of the solvent, the reaction mixture was extracted with chloroform $(30 \mathrm{ml} \times 3)$, and the organic layer was dried over sodium sulfate and concentrated. The residue was treated with column chromatography on silica gel to give cine-substituted product $\mathbf{3 k}$ (eluted with chloroform. $0.20 \mathrm{~g}, 0.51 \mathrm{mmol}, 52 \%$ ) as pale yellow powder $\mathrm{mp}$ $260-261{ }^{\circ} \mathrm{C}$ (dec.). IR $\left(\mathrm{KBr} / \mathrm{cm}^{-1}\right) 1670,1535,1524,1344 ;{ }^{1} \mathrm{H}-\mathrm{NMR}$ $\left(400 \mathrm{MHz}, \mathrm{DMSO}-d_{6}\right) \delta: 2.46(\mathrm{~s}, 3 \mathrm{H}), 3.40(\mathrm{~s}, 3 \mathrm{H}), 4.96(\mathrm{~s}, 2 \mathrm{H}), 6.96(\mathrm{~s}$, $1 \mathrm{H}), 7.39(\mathrm{~d}, J=8.1 \mathrm{~Hz}, 2 \mathrm{H}), 8.02(\mathrm{~d}, J=8.1 \mathrm{~Hz}, 2 \mathrm{H}), 8.71(\mathrm{~d}, J=2.5 \mathrm{~Hz}$, $1 \mathrm{H}), 8.85(\mathrm{~d}, J=2.5 \mathrm{~Hz}, 1 \mathrm{H}) ;{ }^{13} \mathrm{C}-\mathrm{NMR}\left(100 \mathrm{MHz}, \mathrm{DMSO}-d_{6}\right) \delta: 21.2(\mathrm{q})$, 34.3 (q), 41.7 (t), 121.8 (d), 123.6 (s), 124.9 (d), 128.4 (d), 129.1 (d), 133.2 (s), 137.4 (s), 138.5 (s), 139.7 (s), 144.1 (s), 145.2 (s), 161.0 (s), 194.9 (s), One signal was lacked because of overlap. Anal. Calcd for $\mathrm{C}_{19} \mathrm{H}_{15} \mathrm{~N}_{3} \mathrm{O}_{6}$ : C, 59.84; H, 3.97; N, 11.02. Found: C, 59.90; H, 3.84; N, 11.05 .

Reactions of TNQ with other ketones or aldehyde were similarly conducted.

4-(1-Benzoylethyl)-6,8-dinitro-1-methyl-2-oxo-quinoline (3b) Pale yellow needles. $\mathrm{mp} 184-185^{\circ} \mathrm{C}$ (dec.). IR $\left(\mathrm{KBr} / \mathrm{cm}^{-1}\right) 1674,1537,1524$, 1346; ${ }^{1} \mathrm{H}-\mathrm{NMR}\left(400 \mathrm{MHz}, \mathrm{CDCl}_{3}\right) \delta: 1.71(\mathrm{~d}, J=7.0 \mathrm{~Hz}, 3 \mathrm{H}), 3.46(\mathrm{~s}, 3 \mathrm{H})$, $5.15(\mathrm{q}, J=7.0 \mathrm{~Hz}, 1 \mathrm{H}), 6.84(\mathrm{~s}, 1 \mathrm{H}), 7.52(\mathrm{dd}, J=7.4,7.4 \mathrm{~Hz}, 2 \mathrm{H}), 7.64(\mathrm{t}$, $J=7.4 \mathrm{~Hz}, 1 \mathrm{H}), 7.98(\mathrm{~d}, J=7.4 \mathrm{~Hz}, 2 \mathrm{H}), 8.70(\mathrm{~d}, J=2.5 \mathrm{~Hz}, 1 \mathrm{H}) 8.76(\mathrm{~d}$, $J=2.5 \mathrm{~Hz}, 1 \mathrm{H}) ;{ }^{13} \mathrm{C}-\mathrm{NMR}\left(100 \mathrm{MHz}, \mathrm{CDCl}_{3}\right) \delta: 17.2(\mathrm{q}), 34.8$ (q), 43.0 (d), 122.0 (d), 122.7 (d), 122.9 (s), 123.8 (d), 128.5 (d), 129.3 (d), 134.3 (d), 134.9 (s), 138.4 (s), 139.5 (s), 140.3 (s), 148.8 (s), 161.5 (s), 197.8 (s). Anal. Calcd for $\mathrm{C}_{19} \mathrm{H}_{15} \mathrm{~N}_{3} \mathrm{O}_{6} \cdot 1 / 3 \mathrm{H}_{2} \mathrm{O}$ : C, 58.92; $\mathrm{H}, 4.08 ; \mathrm{N}, 10.85$. Found: $\mathrm{C}$, $58.52 ; \mathrm{H}, 3.77 ; \mathrm{N}, 11.13$.

4-( $\alpha$-Benzoylbenzyl)-6,8-dinitro-1-methyl-2-quinolone (3c) Pale yellow needles. mp $249-251^{\circ} \mathrm{C}$ (dec.). IR $\left(\mathrm{KBr} / \mathrm{cm}^{-1}\right) 1680,1662,1543$, 1527,$1346 ;{ }^{1} \mathrm{H}-\mathrm{NMR}\left(400 \mathrm{MHz}, \mathrm{CDCl}_{3}\right) \delta: 3.47(\mathrm{~s}, 3 \mathrm{H}), 6.37(\mathrm{~s}, 1 \mathrm{H}), 6.47$ $(\mathrm{s}, 1 \mathrm{H}), 7.32-7.34(\mathrm{~m}, 2 \mathrm{H}), 7.40-7.51(\mathrm{~m}, 4 \mathrm{H}), 7.63(\mathrm{dd}, J=7.4,7.4 \mathrm{~Hz}$, $2 \mathrm{H}), 8.01-8.03(\mathrm{~m}, 2 \mathrm{H}), 8.57$ (d, $J=2.5 \mathrm{~Hz}, 1 \mathrm{H}), 8.67(\mathrm{~d}, J=2.5 \mathrm{~Hz}, 1 \mathrm{H})$; ${ }^{13} \mathrm{C}-\mathrm{NMR}\left(100 \mathrm{MHz}, \mathrm{CDCl}_{3}\right.$ ) $\delta: 34.9$ (q), 56.2 (d), 121.7 (d), 123.2 (d), 123.4 (s), 126.4 (d), 128.9 (d), 129.1 (d), 129.2 (d), 129.6 (d), 130.0 (d), $133.6(\mathrm{~s}), 134.4(\mathrm{~d}), 135.2(\mathrm{~s}), 138.1(\mathrm{~s}), 139.4(\mathrm{~s}), 140.2$ (s), 147.9 (s), 161.6 (s), $195.6(\mathrm{~s})$

6,8-Dinitro-1-methyl-4-(2-oxocyclopentyl)-2-quinolone (3d) Pale yellow needles. mp $169-170^{\circ} \mathrm{C}$ (dec.). IR $\left(\mathrm{KBr} / \mathrm{cm}^{-1}\right) 1738,1674,1539$, 1520,$1335 ;{ }^{1} \mathrm{H}-\mathrm{NMR}\left(400 \mathrm{MHz}, \mathrm{CDCl}_{3}\right) \delta: 2.11-2.19(\mathrm{~m}, 1 \mathrm{H}), 2.20$ $2.37(\mathrm{~m}, 2 \mathrm{H}), 2.4-2.7(\mathrm{~m}, 3 \mathrm{H}), 3.47(\mathrm{~s}, 3 \mathrm{H}), 3.91(\mathrm{dd}, J=10.8,8.9 \mathrm{~Hz}, 1 \mathrm{H})$, $6.77(\mathrm{~s}, 1 \mathrm{H}), 8.70(\mathrm{~d}, J=2.5 \mathrm{~Hz}, 1 \mathrm{H}), 8.73(\mathrm{~d}, J=2.5 \mathrm{~Hz}, 1 \mathrm{H}) ;{ }^{13} \mathrm{C}-\mathrm{NMR}$ $\left(100 \mathrm{MHz}, \mathrm{CDCl}_{3}\right) \delta: 20.7(\mathrm{t}), 29.3(\mathrm{t}), 34.9(\mathrm{q}), 38.3(\mathrm{t}), 50.9(\mathrm{~d}), 121.9(\mathrm{~d})$, $123.7(\mathrm{~s}), 124.8(\mathrm{~d}), 138.2(\mathrm{~s}), 139.2(\mathrm{~s}), 140.1(\mathrm{~s}), 146.2(\mathrm{~s}), 161.7(\mathrm{~s}), 213.3$ (s), One signal was lacked because of overlap. Anal. Calcd for $\mathrm{C}_{15} \mathrm{H}_{13} \mathrm{~N}_{3} \mathrm{O}_{6} \cdot 1 / 4 \mathrm{H}_{2} \mathrm{O}: \mathrm{C}, 53.65 ; \mathrm{H}, 4.05 ; \mathrm{N}, 12.51$. Found: $\mathrm{C}, 53.46 ; \mathrm{H}, 3.82$; $\mathrm{N}, 12.35$.

6,8-Dinitro-1-methyl-4-(2-methyl-1-oxo-2-propyl)-2-quinolone (3e) Yellow powder. $\mathrm{mp} 157-159^{\circ} \mathrm{C}\left(\mathrm{dec}\right.$.). IR $\left(\mathrm{KBr} / \mathrm{cm}^{-1}\right) 1724,1682,1539$, 1344; ${ }^{1} \mathrm{H}-\mathrm{NMR}\left(400 \mathrm{MHz}, \mathrm{CDCl}_{3}\right) \delta: 1.66(\mathrm{~s}, 6 \mathrm{H}), 3.50(\mathrm{~s}, 3 \mathrm{H}), 7.01(\mathrm{~s}$, $1 \mathrm{H}), 8.47(\mathrm{~d}, J=2.3 \mathrm{~Hz}, 1 \mathrm{H}), 8.71(\mathrm{~d}, J=2.3 \mathrm{~Hz}, 1 \mathrm{H}), 9.73(\mathrm{~s}, 1 \mathrm{H}) ;{ }^{13} \mathrm{C}-\mathrm{NMR}$ $\left(100 \mathrm{MHz}, \mathrm{CDCl}_{3}\right) \delta: 23.1(\mathrm{q}), 35.2(\mathrm{q}), 51.4(\mathrm{~s}), 121.6(\mathrm{~d}), 122.1(\mathrm{~s}), 124.3$ (d), $125.1(\mathrm{~d}), 128.3(\mathrm{~s}), 138.8(\mathrm{~s}), 139.9(\mathrm{~s}), 149.8$ (s), 161.6 (s), 200.5 (d). Anal. Calcd for $\mathrm{C}_{14} \mathrm{H}_{13} \mathrm{~N}_{3} \mathrm{O}_{6} \cdot 1 / 3 \mathrm{H}_{2} \mathrm{O}: \mathrm{C}, 51.70 ; \mathrm{H}, 4.23 ; \mathrm{N}, 12.92$. Found: $\mathrm{C}$, $51.65 ; \mathrm{H}, 3.98 ; \mathrm{N}, 12.58$.

6,8-Dinitro-1-methyl-4-(2-oxo-cyclohexyl)-2-oxo-quinoline (3f) Pale yellow needles. $\mathrm{mp} 229-232{ }^{\circ} \mathrm{C}\left(\mathrm{dec}\right.$.). IR $\left(\mathrm{KBr} / \mathrm{cm}^{-1}\right) 1703,1668,1531$, 1344; ${ }^{1} \mathrm{H}-\mathrm{NMR}\left(400 \mathrm{MHz}, \mathrm{CDCl}_{3}\right) \delta: 1.86-2.01(\mathrm{~m}, 2 \mathrm{H}), 2.11-2.22(\mathrm{~m}$, $2 \mathrm{H}), 2.31-2.35(\mathrm{~m}, 1 \mathrm{H}), 2.40-2.44(\mathrm{~m}, 1 \mathrm{H}), 2.67-2.71(\mathrm{~m}, 2 \mathrm{H}), 3.48(\mathrm{~s}$, $3 \mathrm{H}), 4.10(\mathrm{dd}, J=12.5,4.8 \mathrm{~Hz}, 1 \mathrm{H}), 6.78(\mathrm{~s}, 1 \mathrm{H}), 8.36(\mathrm{~d}, J=2.5 \mathrm{~Hz}, 1 \mathrm{H})$, $8.69(\mathrm{~d}, J=2.5 \mathrm{~Hz}, 1 \mathrm{H}) ;{ }^{13} \mathrm{C}-\mathrm{NMR}\left(100 \mathrm{MHz}, \mathrm{CDCl}_{3}\right) \delta: 25.2(\mathrm{t}), 27.7(\mathrm{t})$, $32.4(\mathrm{t}), 34.9(\mathrm{q}), 42.5(\mathrm{t}), 52.7$ (d), $121.6(\mathrm{~d}), 123.3(\mathrm{~d}), 123.5(\mathrm{~d}), 123.8(\mathrm{~s})$, $138.1(\mathrm{~s}), 139.4(\mathrm{~s}), 140.0(\mathrm{~s}), 147.1(\mathrm{~s}), 161.7$ (s), 207.4 (s). Anal. Calcd for $\mathrm{C}_{16} \mathrm{H}_{15} \mathrm{~N}_{3} \mathrm{O}_{6} \cdot 1 / 4 \mathrm{H}_{2} \mathrm{O}: \mathrm{C}, 54.94 ; \mathrm{H}, 4.47 ; \mathrm{N}, 12.01$. Found: $\mathrm{C}, 54.62 ; \mathrm{H}, 4.20$; $\mathrm{N}, 12.12$.

6,8-Dinitro-1-methyl-4-(2-oxopropyl)-2-quinolone (3g) Pale yellow needles. mp $157-158^{\circ} \mathrm{C}$ (dec.). IR $\left(\mathrm{KBr} / \mathrm{cm}^{-1}\right) 1722,1684,1547,1527$, $1362,1348,1336 ;{ }^{1} \mathrm{H}-\mathrm{NMR}\left(400 \mathrm{MHz}, \mathrm{CDCl}_{3}\right) \delta: 2.43(\mathrm{~s}, 3 \mathrm{H}), 3.49(\mathrm{~s}, 3 \mathrm{H})$, $4.06(\mathrm{~s}, 2 \mathrm{H}), 6.79(\mathrm{~s}, 1 \mathrm{H}), 8.47(\mathrm{~d}, J=2.5 \mathrm{~Hz}, 1 \mathrm{H}), 8.71(\mathrm{~d}, J=2.5 \mathrm{~Hz}, 1 \mathrm{H})$; ${ }^{13} \mathrm{C}-\mathrm{NMR}\left(100 \mathrm{MHz}, \mathrm{CDCl}_{3}\right) \delta: 30.3(\mathrm{q}), 34.9(\mathrm{q}), 47.3(\mathrm{t}), 122.0(\mathrm{~d}), 123.6$ (s), 124.1 (d), 125.8 (d), 138.1 (s), 139.3 (s), 140.3 (s), 142.8 (s), 161.4 (s), 202.2 (s). Anal. Calcd for $\mathrm{C}_{13} \mathrm{H}_{11} \mathrm{~N}_{3} \mathrm{O}_{6}: \mathrm{C}, 51.15 ; \mathrm{H}, 3.63 ; \mathrm{N}, 13.76$. Found: C, $51.00 ; \mathrm{H}, 3.55 ; \mathrm{N}, 13.71$.

6,8-Dinitro-1-methyl-4-(3-oxo-2-butyl)-2-quinolone (3h) and 6,8-Dinitro-1-methyl-4-(2-oxobutyl)-2-quinolone (3i) These products were obtained as a mixture, and their yields were determined by ${ }^{1} \mathrm{H}-\mathrm{NMR} .{ }^{1} \mathrm{H}-\mathrm{NMR}$ $\left(400 \mathrm{MHz}, \mathrm{CDCl}_{3}\right) 3 \mathbf{h} \delta: 1.62(\mathrm{~d}, J=7.0 \mathrm{~Hz}, 3 \mathrm{H}), 2.27(\mathrm{~s}, 3 \mathrm{H}), 3.45(\mathrm{~s}, 3 \mathrm{H})$, $4.23(\mathrm{q}, J=7.0 \mathrm{~Hz}, 1 \mathrm{H}), 6.77(\mathrm{~s}, 1 \mathrm{H}), 8.63(\mathrm{~s}, 2 \mathrm{H}) ; 3 \mathbf{i} \delta: 1.15(\mathrm{t}, J=7.0 \mathrm{~Hz}$, $3 \mathrm{H}), 2.71$ (q, $J=7.0 \mathrm{~Hz}, 2 \mathrm{H}), 3.45$ (s, 3H), 4.03 (s, 2H), $6.71(\mathrm{~s}, 1 \mathrm{H}), 8.63$ (s, $2 \mathrm{H})$ 
6,8-Dinitro-1-methyl-4-(3-oxo-2-pentyl)-2-quinolone (3j) Yellow oil IR $\left(\mathrm{KBr} / \mathrm{cm}^{-1}\right) 1716,1682,1539,1348 ;{ }^{1} \mathrm{H}-\mathrm{NMR}\left(400 \mathrm{MHz}, \mathrm{CDCl}_{3}\right) \delta$ : $1.10(\mathrm{t}, J=7.2 \mathrm{~Hz}, 3 \mathrm{H}), 1.63(\mathrm{~d}, J=7.0 \mathrm{~Hz}, 3 \mathrm{H}), 2.64(\mathrm{q}, J=7.2 \mathrm{~Hz}, 2 \mathrm{H}), 3.48$ $(\mathrm{s}, 3 \mathrm{H}), 4.31(\mathrm{q}, J=7.0 \mathrm{~Hz}, 1 \mathrm{H}), 6.84(\mathrm{~s}, 1 \mathrm{H}), 8.73(\mathrm{~s}, 2 \mathrm{H}) ;{ }^{13} \mathrm{C}-\mathrm{NMR}$ $\left(100 \mathrm{MHz}, \mathrm{CDCl}_{3}\right) \delta: 7.9(\mathrm{q}), 16.2(\mathrm{q}), 34.3(\mathrm{t}), 34.9(\mathrm{q}), 48.0(\mathrm{~d}), 121.9(\mathrm{~d})$, $123.2(\mathrm{~s}), 123.2$ (d), 123.2 (d), 138.2 (s), 139.4 (s), 140.2 (s), 148.1 (s), $161.5(\mathrm{~s}), 208.4(\mathrm{~s})$

4-(3,4-Benzo-2-oxocyclohexyl)-6,8-dinitro-1-methyl-2-quinolone $(3 \mathrm{~m})$ Pale orange needles. mp $227-229^{\circ} \mathrm{C}$ (dec.). IR $\left(\mathrm{KBr} / \mathrm{cm}^{-1}\right) 1675,1539$, 1525,$1340 ;{ }^{1} \mathrm{H}-\mathrm{NMR}\left(400 \mathrm{MHz}, \mathrm{CDCl}_{3}\right) \delta: 2.52$ (dddd, $J=12.7,4.1,4.1$, $3.8 \mathrm{~Hz}, 1 \mathrm{H}$ ), 2.63 (dddd, $J=12.7,12.7,12.0,4.0 \mathrm{~Hz}, 1 \mathrm{H}$ ), 3.44 (ddd, $J=16.7$, $3.8,4.0 \mathrm{~Hz}, 1 \mathrm{H}), 3.35$ (ddd, $J=16.7,12.0,4.1 \mathrm{~Hz}, 1 \mathrm{H}), 3.49$ (s, $3 \mathrm{H}), 4.33$ (dd, $J=12.7,4.1 \mathrm{~Hz}, 1 \mathrm{H}), 6.81(\mathrm{~s}, 1 \mathrm{H}), 7.35-7.41(\mathrm{~m}, 2 \mathrm{H}), 7.60(\mathrm{t}$, $J=6.8 \mathrm{~Hz}, 1 \mathrm{H}), 8.03(\mathrm{~d}, J=7.6 \mathrm{~Hz}, 1 \mathrm{H}), 8.59(\mathrm{~d}, J=2.4 \mathrm{~Hz}, 1 \mathrm{H}), 8.70(\mathrm{~d}$, $J=2.4 \mathrm{~Hz}, 1 \mathrm{H}) ;{ }^{13} \mathrm{C}-\mathrm{NMR}\left(100 \mathrm{MHz}, \mathrm{CDCl}_{3}\right) \delta: 27.9(\mathrm{t}), 29.3(\mathrm{t}), 35.0(\mathrm{q})$, 50.0 (d), 121.7 (d), 123.0 (d), 124.2 (s), 124.2 (d), 127.3 (d), 128.1 (d), 129.0 (d), 131.8 (s), 134.6 (d), 138.0 (s), 139.3 (s), 140.2 (s), 143.5 (s), 148.3 (s), 161.7 (s), 194.9 (s). Anal. Calcd for $\mathrm{C}_{20} \mathrm{H}_{15} \mathrm{~N}_{3} \mathrm{O}_{6}:$ C, 61.07; H, $3.84 ; \mathrm{N}, 10.69$. Found: C, 59.83; H, 3.73; N, 10.28.

6,8-Dinitro-1-methyl-4-\{2-oxo-2-(2-pyridyl)ethyl\}-2-quinolone (3n) Pale orange needles. mp. $201-203^{\circ} \mathrm{C}$ (dec.). IR $\left(\mathrm{KBr} / \mathrm{cm}^{-1}\right) 1695,1680$, 1538, 1340; ${ }^{1} \mathrm{H}-\mathrm{NMR}\left(400 \mathrm{MHz}, \mathrm{CDCl}_{3}\right.$ ) $\delta: 3.49$ (s, 3H), 4.89 (s, 2H), 6.96 (s, $1 \mathrm{H}), 7.62$ (ddd, $J=7.7,4.7,0.9 \mathrm{~Hz}, 1 \mathrm{H}), 7.93$ (ddd, $J=7.7,7.7,1.7 \mathrm{~Hz}$, $1 \mathrm{H}), 8.08$ (dd, $J=7.7,0.9 \mathrm{~Hz}, 1 \mathrm{H}), 8.70(\mathrm{~d}, J=2.5 \mathrm{~Hz}, 1 \mathrm{H}), 8.83$ (dd, $J=4.7$, $1.7 \mathrm{~Hz}, 1 \mathrm{H}), 8.90(\mathrm{~d}, J=2.5 \mathrm{~Hz}, 1 \mathrm{H}) ;{ }^{13} \mathrm{C}-\mathrm{NMR}\left(100 \mathrm{MHz}, \mathrm{CDCl}_{3}\right) \delta: 34.9$ (q), 40.8 (t), 121.9 (d), 122.7 (d), 124.0 (s), 124.7 (d), 126.3 (d), 128.4 (d), 137.5 (d), 138.1 (s), 139.2 (s), 140.3 (s), 144.2 (s), 149.4 (d), 151.8 (s), 161.5 (s), 196.4 (s). Anal. Calcd for $\mathrm{C}_{17} \mathrm{H}_{12} \mathrm{~N}_{4} \mathrm{O}_{6}: \mathrm{C}, 55.44 ; \mathrm{H}, 3.28 ; \mathrm{N}$, 15.21. Found: C, 55.49; H, 3.19; N, 15.15 .

6,8-Dinitro-1-methyl-4-\{2-oxo-2-(2-furyl)ethyl\}-2-quinolone (3o) Pale yellow needles. mp. $212-214^{\circ} \mathrm{C}$ (dec.). IR $\left(\mathrm{KBr} / \mathrm{cm}^{-1}\right) 1670,1541$, $1335 ;{ }^{1} \mathrm{H}-\mathrm{NMR}\left(400 \mathrm{MHz}, \mathrm{CDCl}_{3}\right) \delta: 3.49(\mathrm{~s}, 3 \mathrm{H}), 4.47(\mathrm{~s}, 2 \mathrm{H}), 6.68(\mathrm{dd}$, $J=3.6,1.7 \mathrm{~Hz}, 1 \mathrm{H}), 6.92(\mathrm{~s}, 1 \mathrm{H}), 7.39(\mathrm{dd}, J=3.6,0.6 \mathrm{~Hz}, 1 \mathrm{H}), 7.73(\mathrm{dd}$, $J=1.7,0.6 \mathrm{~Hz}, 1 \mathrm{H}), 8.72(\mathrm{~d}, J=2.5 \mathrm{~Hz}, 1 \mathrm{H}), 8.73(\mathrm{~d}, J=2.5 \mathrm{~Hz}, 1 \mathrm{H}) ;{ }^{13} \mathrm{C}-$ NMR $\left(100 \mathrm{MHz}, \mathrm{CDCl}_{3}\right) \delta: 34.4$ (q), 42.0 (t), 113.4 (d), 118.9 (d), 122.0 (d), 123.8 (s), 124.5 (d), 126.3 (d), 138.1 (s), 139.2 (s), 140.3 (s), 142.8 (s), 147.5 (d), 151.6 (s), 161.4 (s), 182.9 (s). Anal. Calcd for $\mathrm{C}_{16} \mathrm{H}_{11} \mathrm{~N}_{3} \mathrm{O}_{7}$ : C, $53.79 ; \mathrm{H}, 3.10 ; \mathrm{N}, 11.76$. Found: C, 52.76; H, 2.91; N, 11.65 .

\section{References}

1) Ito C., Nat. Med., 54, 117-122 (2000).

2) Grundon M. F., Nat. Prod. Rep., 7, 131-138 (1990).

3) Grundon M. F., "The Alkaloids: Quinoline Alkaloids Related to Anthranic Acid," Vol. 32, Academic Press, London, 1968, pp. 341- 439.

4) Klasek A., Koristek K., Sedmera P., Halada P., Heterocycles, 60, 799815 (2003).

5) Kumar R. N., Selvi S. T., Suresh T., Mohan P. S., Heterocycles, 57, $357-360$ (2002).

6) McLaughlin M. J., Hsung R, P., J. Org. Chem., 66, 1049-1053 (2001).

7) Lee Y. R., Kweon H. I., Koh W. S., Min K. R., Kim Y., Lee S. H., Synthesis, 2001, 1851-1855 (2001).

8) Bar G., Parsons A. F., Thomas C. B., Tetrahedron, 57, 4719-4728 (2001).
9) Boyd D. R., Sharma N. D., Barr S. A., Caroll J. G., Mackerracher D., Maolone J. F., J. Chem. Soc., Perkin Trans. 1, 2000, 3397-3405 (2000).

10) Ito C., Kondo Y., Wu T.-S., Furukawa H., Chem. Pharm. Bull., 48 , 65-70 (2000).

11) Kamperdick C., Van N., Sung T. V., Adam G., Phytochemistry, 50, 177-181 (1999).

12) Michael J. P., Nat. Prod. Rep., 16, 697-709 (1999).

13) Atta-ur-Rahman, Sultana N., Choudhary M. I., Shah P. M., Khan M. R., J. Nat. Prod., 61, 713-717 (1998).

14) Chen I.-S., Tsai I.-W., Teng C.-M., Chen J.-J., Chen J.-J., Chang Y.-L., Ko F.-N., Lu M. C., Pezzuto J. M., Phytochemistry, 46, 525-529 (1997).

15) Brader G., Bacher M., Greger H., Hofer O., Phytochemistry, 42, 881884 (1996).

16) Kumabe R., Nishino H., Tetrahedron Lett., 45, 703-706 (2004).

17) Majumdar K. C., Mukhopadhyay P. P., Synthesis, 2003, 97-100 (2003).

18) Täubl A. E., Langhans K., Kappe T., Stadlbauer W., J. Heterocycl. Chem., 39, 1259-1264 (2002).

19) Nair V., Vinod A. U., Ramesh R., Menon R. S., Varma L., Mathew S., Chiaroni A., Heterocycles, 58, 147-151 (2002).

20) Ghosh M., Jana M., Synthesis, 2002, 669-673 (2002).

21) Bar G., Parsons A. F., Thomas C. B., Chem. Commun., 2001, 13501351 (2001).

22) Lee Y. R., Suk J. Y., Kim B. S., Org. Lett., 2, 1387-1389 (2000)

23) Hojas G., Fiala W., Stadlbauer W., J. Heterocycl. Chem., 37, 15591569 (2000).

24) Pirrung M. C., Blume F., J. Org. Chem., 64, 3642-3649 (1999).

25) Ye J.-H., Ling K.-Q., Zhang Y., Li N., Xu J.-H., J. Chem. Soc., Perkin Trans. 1, 1999, 2017-2023 (1999).

26) Majumdar K. C., Kundu A. K., Biswas P., Heterocycles, 51, 23992406 (1999).

27) Toche R. B., Jachak M. N., Sabnis R. W., Kappe T., J. Heterocycl. Chem., 36, 467-471 (1999).

28) Mitsos C., Petrou J., Igglessi-Markopoulou O., J. Heterocycl. Chem., 36, 881-887 (1999).

29) Tagawa Y., Kawaoka T., Goto Y., J. Heterocycl. Chem., 34, 16771683 (1997).

30) Fujita R., Oikawa K., Yoshisuji T., Okuyama Y., Nakano H., Matsuzaki H., Chem. Pharm. Bull., 51, 295-300 (2003).

31) Fujita R., Watanabe K., Yoshisuji T., Kabuto C., Matsuzaki H., Hongo H., Chem. Pharm. Bull., 49, 893-899 (2001).

32) Fujita R., Watanabe K., Yoshisuji T., Hongo H., Matsuzaki H., Chem. Pharm. Bull., 49, 900-904 (2001).

33) Nishiwaki N., Tanaka A., Uchida M., Tohda Y., Ariga M., Bull. Chem. Soc. Jpn., 69, 1377-1381 (1996).

34) Nishiwaki N., Tanaka C., Asahara M., Asaka N., Tohda Y., Ariga M., Heterocycles, 51, 567-574 (1999).

35) Nishiwaki N., Sakashita M., Azuma M., Tanaka C., Tamura M., Asaka N., Hori K., Tohda Y., Ariga M., Tetrahedron, 58, 473-478 (2002).

36) Prill E. A., McElvain S. M., Org. Synth., Coll. Vol. 2, 419-421 (1943). 